\title{
A novel damage detection algorithm using time-series analysis-based blind source separation
}

\author{
A. Sadhu* and B. Hazra \\ Department of Civil and Environmental Engineering, University of Waterloo, Waterloo, ON, Canada
}

Received 17 August 2012

Revised 6 November 2012

Accepted 9 November 2012

\begin{abstract}
In this paper, a novel damage detection algorithm is developed based on blind source separation in conjunction with time-series analysis. Blind source separation (BSS), is a powerful signal processing tool that is used to identify the modal responses and mode shapes of a vibrating structure using only the knowledge of responses. In the proposed method, BSS is first employed to estimate the modal response using the vibration measurements. Time-series analysis is then performed to characterize the mono-component modal responses and successively the resulting time-series models are utilized for one-step ahead prediction of the modal response. With the occurrence of newer measurements containing the signature of damaged system, a variance-based damage index is used to identify the damage instant. Once the damage instant is identified, the damaged and undamaged modal parameters of the system are estimated in an adaptive fashion. The proposed method solves classical damage detection issues including the identification of damage instant, location as well as the severity of damage. The proposed damage detection algorithm is verified using extensive numerical simulations followed by the full scale study of UCLA Factor building using the measured responses under Parkfield earthquake.
\end{abstract}

Keywords: Blind source separation, time series analysis, damage detection, ambient modal identification, damage index

\section{Introduction}

Blind Source Separation (BSS) methods have recently emerged as a powerful class of signal processing methods capable of monitoring the health of a large class of civil structures. The application of BSS methods to flexible structures have been reported for a broad range of numerical and experimental studies, e.g., $[2,10,11,13,19]$. These results clearly reveal the potential of using the principle of BSS for a wide range of structural engineering problems. Originally proposed for a fewer class of problems involving broad-band excitations, static mixtures, and relatively large sensor densities, BSS extensions to under-determined case [12,14], nonstationary environment [31], de-centralized sensing network [29,32], and for convolutive mixing [30] have also been reported in recent studies by the authors. In the present study, a novel time-series analysis based BSS method is proposed to tackle damage detection in civil structures which is commonly encountered as a major structural health monitoring problem.

Structural health monitoring (SHM) entails development and implementation of damage diagnosis and prognosis algorithms for accurate economical management and maintenance of modern civil infrastructures. Relatively

\footnotetext{
${ }^{*}$ Corresponding author: A. Sadhu, Department of Civil and Environmental Engineering, University of Waterloo, Waterloo, ON N2L 3G1, Canada. E-mail: ayansadhu.civil@gmail.com.
} 


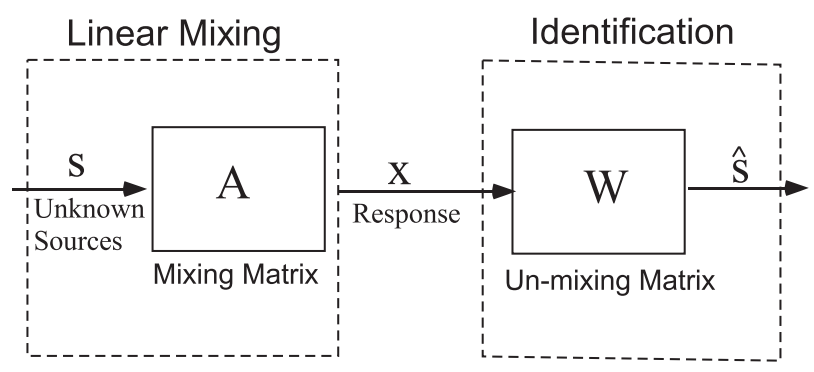

Fig. 1. Basic framework of blind source separation.

vast literature [6,9] presents an extensive survey of various damage detection techniques in mechanical and civil structures that utilize changes in modal properties such as natural frequency, damping and mode shapes. Damage indicators are primarily based on changes in the modal parameters that reflect equivalent variations in the physical parameters of the system. Such alterations in the physical parameters commonly occur due to structural degradation under various environmental conditions, human-induced excitation or natural events such as earthquake and strong winds. Recently blind source separation (BSS), a powerful signal processing tool, has gained significant attentions in the field of structural system identification [2,14,29]. Such BSS-based modal identification methods have shown significant promises in either mild non-stationary ambient vibration [12,29] or severe non-stationary event like earthquake excitation [31]. In the present paper, an adaptive damage detection algorithm is developed in the framework of blind source separation in conjunction with time-series analysis.

Various damage detection techniques [6,36] are developed in the last decades, which can be broadly classified based on the level of attempted identification: (a) presence, (b) location, (c) severity of damage [28]. Consequently, recent development in signal processing tools [36] has resulted a paradigm shift in its application towards damage detection, leading to a class of algorithms employing various time-frequency transformation based approaches using wavelets [16,34], empirical mode decomposition and Hilbert-Huang transform [37], statistical methods like principal component analysis [26,35] and time-series models [4,7,22,24,38]. Recently, another powerful signal processing method based on blind source separation principles (BSS) [2], has been studied in the context of structure modal identification $[2,12,14,29,31]$. These methods show excellent applicability to the problem of structural mode identification, and are explored here to the case of damage detection.

The basic problem statement of BSS as illustrated in Fig. 1, is given by:

$$
\begin{aligned}
& \mathbf{x}(n)=\mathbf{A} \mathbf{s}(n) \\
& \hat{\mathbf{s}}(n)=\mathbf{y}(n)=\mathbf{W} \mathbf{x}(n)
\end{aligned}
$$

where, $\mathbf{A}_{n_{m} \times n_{s}}$ is the instantaneous mixing matrix and $\mathbf{W}_{n_{s} \times n_{m}}$ is the estimated un-mixing matrix, which is the inverse of $\mathbf{A}$ for the case when the number of sources $\left(n_{s}\right)$ is equal to the number of measurements $\left(n_{m}\right)$. An estimate of the sources $\hat{\mathbf{s}}$ are then given by $\mathbf{y}$. BSS methods attempt to determine the un-mixing matrix $\mathbf{W}$ and $\hat{\mathbf{s}}$ using the information contained in $\mathrm{x}$ only. Hence, the term blind is commonly used. There are two popular BSS approaches in the literature: higher-order statistics (HOS) based independent component analysis (ICA) [17], and second-order statistics (SOS) based second-order blind identification (SOBI) [3]. ICA exploits the marginal non-Gaussianity and statistical independence of sources without considering the time structure of sources. On the other hand, SOBI utilizes SOS by constructing multiple co-variance matrices of the responses, for the case when the sources are spatially uncorrelated but temporally correlated [8]. The concept of BSS has been extended to the ambient system identification of structural systems [2,12,14,29,31], where the normal modes of a linear dynamical system can be regarded as virtual sources. Recently ICA-based BSS method [18,27,39] is developed for the structural damage detection. However, the ICA methods suffer performance issues in presence of structural damping even in the order of $2 \%$ [11]. Unlike ICA, SOBI-based BSS method that considers time structure pertaining to several time-lagged co-variance matrices, enables better handling of sources with different spectral contents. In the present study, SOBI-based BSS method is proposed in conjunction with time-series analysis to detect the damage in the structural systems.

The effects of damage in a structure are classified as linear or nonlinear [9]. A linear damage situation is defined as the case when a initially linear-elastic structure remains linear-elastic after damage. Such elastic changes in 
modal properties primarily occur due to changes in the geometry and/or the material properties of the structure, however the structural response can still be modeled using a linear equation of motion [9]. This is the basis of the proposed method where the SOBI-based BSS is used for the modal identification, and then the estimated sources in conjunction with the time-series analysis are used for the prediction of future measurements to identify the damage. Time-series models $[5,15]$ are used to characterize the sources that are obtained from the past observations and then the future sources (i.e., the measurements) are predicted. With the arrival of newer measurements containing the signature of structural damage, significant difference is observed between the predicted and true measurements, which is used as a damage indicator. Once the damage is detected, the measurements prior to the damage are discarded and the modal parameters of the damaged state are estimated based on the current measurements. In this way, the damage and un-damaged modal parameters are estimated in an adaptive fashion. The proposed method is used to solve the previously mentioned three classes of damage detection problems including identification of presence, location and severity of damage.

This paper is organized as follows. The problem statement is presented first wherein the general problem of structural system identification is cast in BSS framework followed by the verification using the extensive numerical studies. Finally, the identification results of the UCLA building are presented, followed by the main conclusions of this study.

\section{Background}

The algorithm developed in this paper is based on the concept of prediction of sources of the BSS method using time-series analysis. The sources are first estimated based on the current measurements using the BSS method, which are then modeled by suitable time-series models and are used to predict the future measurements. With the arrival of true future measurements, an error is evaluated between the true and predicted measurements and subsequently the damage is identified. Instead of time-series modeling of raw vibration measurements, the estimated sources are chosen for the prediction of the measurements. This is primarily due to complexity in developing timeseries models from raw vibration measurements containing large number of degrees-of-freedom with significant measurement noise [7,22,24]. On the other hand, mono-component sources are easy to predict using low-order time-series model [25] and thus the accurate response prediction is obtained for the damage detection. This is the essence of the proposed method. A brief background of time-series analysis is included herein.

\subsection{Time-series model}

In general, an auto-regressive moving average (ARMA) $(p, q)$ model [5,15] for a zero-mean time series $(x)$ can be described as:

$$
x_{t}-\phi_{1} x_{t-1}-\phi_{2} x_{t-2}-\ldots-\phi_{p} x_{t-p}=a_{t}-\theta_{1} a_{t-1}-\theta_{2} a_{t-2}-\ldots-\theta_{q} a_{t-q}
$$

Using $B$ operator, Eq. (2) can be expressed as:

$$
\begin{aligned}
& \left(1-\phi_{1} B-\phi_{2} B^{2}-\ldots-\phi_{p} B^{p}\right) x_{t}=\left(1-\theta_{1} B-\theta_{2} B^{2}-\ldots-\theta_{q} B^{q}\right) a_{t} \\
& \phi(B) x_{t}=\theta(B) a_{t}
\end{aligned}
$$

where $\phi(B)$ and $\theta(B)$ contain auto-regressive (AR) and moving average (MA) parameters respectively. $a_{t}$ is the zero-mean white noise sequence characterized by its variance, $\sigma_{a}^{2}$. The AR parameters of order $p$ describe the system dynamics while MA part of order $q$ is related to external disturbances, and ensures the overall stationarity of the response. An appropriate time series model is primarily developed using three steps process consisting of (a) identification, (b) parameter estimation and (c) diagonastic checks [5,15]. In order to identify the model order (i.e., $p$ and $q$ ), several forms of auto-correlation functions (ACF) are used such as partial auto-correlation function (PACF), inverse auto-correlation function (IACF) and inverse partial auto-correlation function (IPACF). Sample ACF $\left(r_{k}\right)$ is defined as [15]:

$$
c_{k}=\frac{1}{N} \sum_{i=1}^{N-k}\left(x_{i}-\bar{x}\right)\left(x_{i-k}-\bar{x}\right)
$$



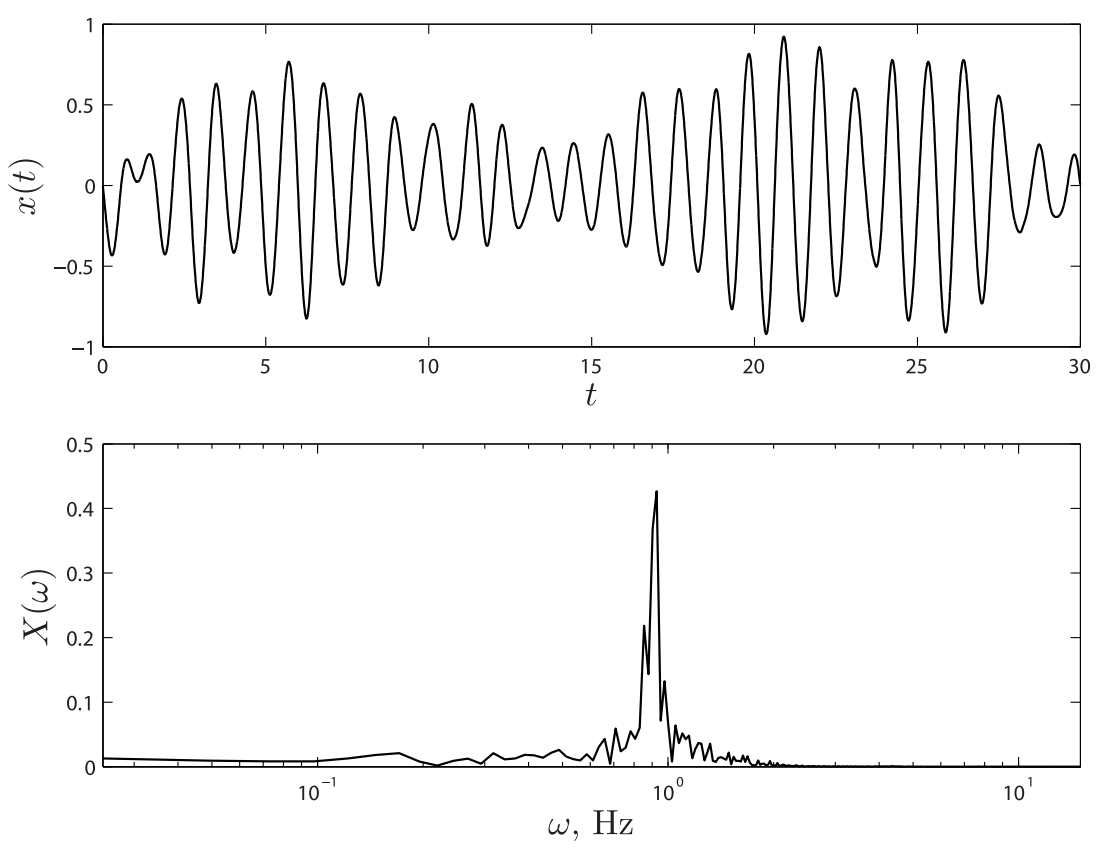

Fig. 2. Response of a SDOF system and its Fourier spectrum.

$$
r_{k}=\frac{c_{k}}{c_{0}}
$$

The PACF is defined as a function which cuts off for an AR process. Sample PACF, $\phi_{k j}$ is defined as the $j^{\text {th }}$ coefficient in a stationary AR process of order $k$ so that $\phi_{k k}$ is the last coefficient. It can be calculated using YuleWalker equation [15]. IACF is defined as the ACF associated with the reciprocal of the spectral density function and is expressed as [15]:

$$
r i_{k}=\frac{\hat{\phi}_{k}+\sum_{i=1}^{r-k} \hat{\phi}_{i} \hat{\phi}_{i+k}}{1+\sum_{i=1}^{r} \hat{\phi}_{i}^{2}} \quad 10<r<40
$$

The characteristics of IACF are similar to PACF. The inverse PACF $\left(\theta_{k k}\right)$ is the PACF of an ARMA $(q, p)$ process [15]. One can replace the sample IACF in the Yule-Walker equation and solve for the IPACF. The characteristics of IPACF are similar to ACF. Once the mode order is decided, the model parameters are estimated using Yule-Walker equations. The chosen model order is then optimized using Akaike information criteria (AIC) [1]. When there are several suitable models available for a time series, the model possessing minimum value of AIC is selected.

In order to demonstrate the use of time series analysis in the present context, a simple example is shown herein. Figure 2 shows the response of a single-degree-of-freedom (SDOF) system under white noise excitation and its Fourier spectra respectively. Such SDOF system simulates the mono-component sources that are obtained from BSS. The sample ACF, PACF, IACF and IPACF of the response are shown in Fig. 3. It can be observed that the ACF trends to follow a damped exponential curve, which suggests that some sort of AR models fit the data [15]. The sample PACF and IACF cut off after lag $k=2$, which suggests that AR(2) type of model would be suitable for the data. The slow decay of sample IPACF also indicates the presence of single frequency in the data and confirms the suitability of the low order AR model. The results are summarized in Table 1. It is noted that the sources extracted from BSS are primarily mono-component signal, and therefore can be modeled using AR(2) model. Once the model order is selected, the associated AR coefficients $\phi(B)$ are estimated using Yule-Walker equation [15]. Such chosen AR(2) model can be used for minimum mean-square error forecast of measurement $x_{t}$ using Eq. (2) with the following expression $[5,15]$ :

$$
\hat{x}_{t}=\phi_{1} x_{t-1}+\phi_{2} x_{t-2}+a_{t}
$$

where $\phi_{i}$ is the $i^{\text {th }}$ AR coefficient. 
Table 1

Identification results of $x(t)$

\begin{tabular}{llll}
\hline$x(t)$ & Fig. & \multicolumn{1}{c}{ Observations } & Proposed model \\
\hline Time-series & 2 & Zero-mean stationary process & Stationary \\
ACF & 3(a) & Attenuates & $\operatorname{AR}(p) / \operatorname{ARMA}(p, q)$ \\
PACF & 3(b) & Truncates at lag 2 & $\operatorname{AR}(2)$ \\
IACF & 3(c) & Truncates at lag 2 & $\operatorname{AR}(2)$ \\
IPACF & 3(d) & Attenuates & $\operatorname{AR}(p) / \operatorname{ARMA}(p, q)$ \\
\hline
\end{tabular}

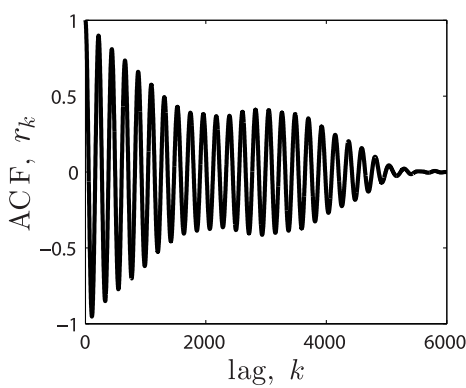

(a)

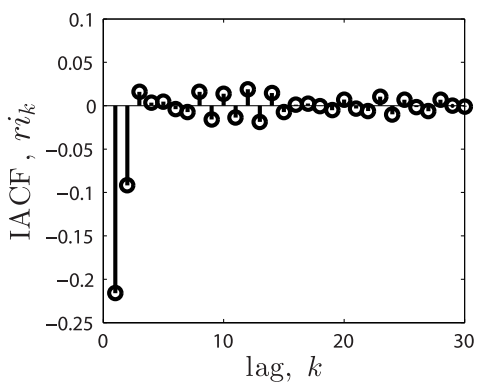

(c)

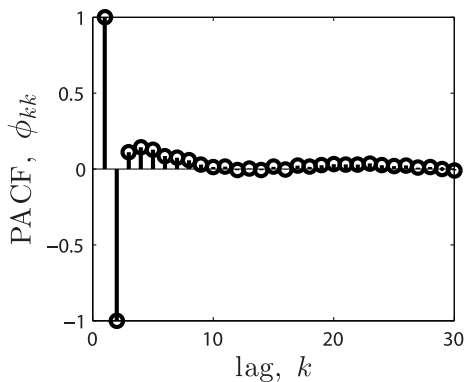

(b)

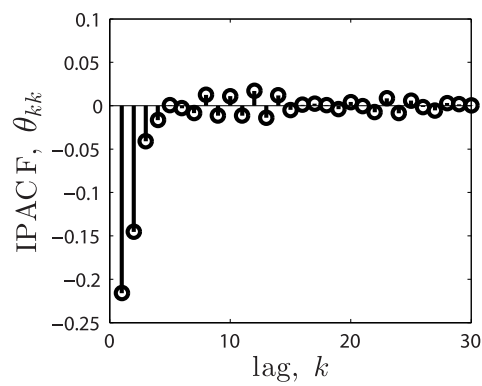

(d)

Fig. 3. (a) ACF, (b) PACF, (c) IACF, (d) IPACF of $x(t)$.

\section{Proposed method}

Consider a linear, classically damped, and lumped-mass $n_{s}$ degrees-of-freedom structural system, subjected to an excitation force, $\mathbf{F}(t)$.

$$
\mathbf{M} \ddot{\mathbf{x}}(t)+\mathbf{C} \dot{\mathbf{x}}(t)+\mathbf{K} \mathbf{x}(t)=\mathbf{F}(t)
$$

where, $\mathbf{x}(t)$ is a vector of displacement coordinates at the degrees of freedom. $\mathbf{M}, \mathbf{C}$, and $\mathbf{K}$ are the mass, damping and stiffness matrices of the multi-degree-of-freedom system. For the case of earthquake excitation (ground acceleration, $\left.\ddot{u}_{g}(t)\right), \mathbf{F}(t)=-\mathbf{M I} \ddot{u}_{g}(t)$ ( $\mathbf{I}$ is the identity matrix), the solution to Eq. (7) can be written in terms of superposition of vibration modes with the following matrix form:

$$
\mathbf{x}=\Psi \mathbf{q}
$$

where, $\mathbf{x} \in \Re^{n_{m} \times N}$ is the trajectory matrix composed of the sampled components of $\mathbf{x}, \mathbf{q} \in \Re^{n_{s} \times N}$ is a matrix of the corresponding modal coordinates, $\Psi_{n_{m} \times n_{s}}$ is the modal transformation matrix, and $N$ is the number of data points of the measurements. The measurement at $i^{\text {th }}$ degree-of-freedom $\left(i=1,2, \ldots n_{m}\right)$ of Eq. (8) can be expressed as $x_{i}(t)=\sum_{r=1}^{n_{s}} \psi_{i r} q_{r}(t)$ with

$$
q_{r}(t)=\frac{\mu_{r}}{M_{r} \omega_{r}} W_{r}(t)
$$


where

$$
W_{r}(t)=\int_{0}^{T} \ddot{u}_{g}(\tau) e^{-\tau_{r} \omega_{r}(t-\tau)} \sin \omega_{r}(t-\tau) d \tau ; \quad M_{r}=\psi_{r}^{T} \mathbf{M} \psi_{r} ; \quad \mu_{r}=\psi_{r}^{T} \mathbf{M I}
$$

Note the similarity between Eqs (1) and (8). Under the conditions where the modal coordinates are mutually uncorrelated with non-similar spectra, the normal coordinates can be regarded as the most uncorrelated sources. Thus, the modal coordinates $\mathbf{q}$ are a special case of general sources $\mathbf{s}$ with time structure, and subsequently form the basis of the BSS-based modal identification procedure described in this paper. Furthermore, the scalar multiplication for the components in $\mathrm{x}$ is not expected to introduce ambiguity in the process of modal identification.

The proposed method operates under the basic framework of SOBI, resulting in the following steps: simultaneous diagonalization of two covariance matrices $\mathbf{R}_{\mathbf{x}}(0)$ and $\mathbf{R}_{\mathbf{x}}(p)$ evaluated at the time-lag zero and $p$, respectively. This can be written as:

$$
\begin{aligned}
& \mathbf{R}_{\mathbf{x}}(0)=E\left\{\mathbf{x}(n) \mathbf{x}^{T}(n)\right\}=\mathbf{A R}_{\mathbf{s}}(0) \mathbf{A}^{T} \\
& \mathbf{R}_{\mathbf{x}}(p)=E\left\{\mathbf{x}(n) \mathbf{x}^{T}(n-p)\right\}=\mathbf{A R}_{\mathbf{s}}(p) \mathbf{A}^{T}
\end{aligned}
$$

where,

$$
\mathbf{R}_{\mathbf{s}}(p)=E\left\{\mathbf{s}(n) \mathbf{s}^{T}(n-p)\right\}
$$

The following three steps set up the essence of SOBI: whitening, orthogonalization, and unitary transformation. Whitening is a linear transformation in which, $\mathbf{R}_{\mathbf{x}}(0)=(1 / N)\left(\sum_{n=1}^{N} \mathbf{x}(n) \mathbf{x}^{T}(n)\right)$ is first diagonalized using singular value decomposition, $\mathbf{R}_{\mathbf{x}}(0)=\mathbf{V}_{\mathbf{x}} \boldsymbol{\Lambda}_{\mathbf{x}} \mathbf{V}_{\mathbf{x}}^{T}$ where $\boldsymbol{\Lambda}_{\mathbf{x}}$ and $\mathbf{V}_{\mathbf{x}}$ are the eigenvalues and eigenvectors of the co-variance matrix of $\mathbf{R}_{\mathbf{x}}(0)$ respectively. Then, the standard whitening is realized by a linear transformation expressed as,

$$
\overline{\mathbf{x}}(n)=\mathbf{Q} \mathbf{x}(n)=\mathbf{\Lambda}_{\mathbf{x}}^{-\frac{1}{2}} \mathbf{V}_{\mathbf{x}}^{T} \mathbf{x}(n)
$$

Because of whitening, $\mathbf{R}_{\mathbf{x}}(p)$ becomes $\mathbf{R}_{\overline{\mathbf{x}}}(p)$, which is given by the equation,

$$
\mathbf{R}_{\overline{\mathbf{x}}}(p)=\left(\frac{1}{N}\right)\left(\sum_{n=1}^{N} \overline{\mathbf{x}}(n) \overline{\mathbf{x}}^{T}(n-p)\right)=\mathbf{Q} \mathbf{R}_{\mathbf{x}}(p) \mathbf{Q}^{T}
$$

Using the Eqs (11) and (14), we get

$$
\mathbf{R}_{\overline{\mathbf{x}}}(p)=\mathbf{Q} \mathbf{A} \mathbf{R}_{\mathbf{s}}(p) \mathbf{A}^{T} \mathbf{Q}^{T}
$$

The above equation states that by diagonalizing the whitened covariance matrix at a particular time-lag, the unitary matrix product QA can be determined, resulting in the mixing matrix, A. This process of diagonalization is implemented numerically, and typically involves jointly diagonalizing several covariance matrices at a given lag $p$ [12]. The second step, called orthogonalization, is applied to diagonalize the matrix $\mathbf{R}_{\overline{\mathbf{x}}}(p)$ whose eigen-value decomposition satisfies

$$
\mathbf{V}_{\overline{\mathbf{x}}} \mathbf{R}_{\overline{\mathbf{x}}}(p) \mathbf{V}_{\overline{\mathbf{x}}}^{T}=\boldsymbol{\Lambda}_{\overline{\mathbf{x}}}
$$

Since the diagonal matrix $\boldsymbol{\Lambda}_{\overline{\mathbf{x}}}$ has distinct eigen-values, it is easy to see that the product $\mathbf{Q A}$ is a unitary matrix, and the mixing matrix can be estimated by the equation,

$$
\hat{\mathbf{A}}=\mathbf{Q}^{-1} \mathbf{V}_{\overline{\mathbf{x}}}=\mathbf{V}_{\mathbf{x}} \Lambda_{\mathbf{x}}^{1 / 2} \mathbf{V}_{\overline{\mathbf{x}}}
$$

where $\hat{\mathbf{A}}$ is the estimated mixing matrix of $\mathbf{A}$. The problem now becomes one of unitary diagonalization of the correlation matrix $\mathbf{R}_{\overline{\mathbf{x}}}(p)$ at one or several non-zero time lags. Equation (15) is a key result, which states that the whitened covariance matrix $\mathbf{R}_{\overline{\mathbf{x}}}(p)$ at any non-zero time lag $p$ is diagonalized by the unitary matrix $\mathbf{Q A}$. The determination of the unitary matrix is carried out using a numerical procedure, commonly known as joint approximate diagonalization [3]. Denoting $\mathbf{V}=\mathbf{Q A}, \mathbf{D}=\mathbf{V}^{T} \widetilde{\mathbf{R}}_{\overline{\mathbf{x}}}(p) \mathbf{V}$, the problem is one of finding the minimum of the performance index $J$ given by,

$$
J(\mathbf{V}, \mathbf{p})=\sum_{p} \sum_{1 \leqslant i \neq j \leqslant n_{s}}\left|D_{i j}^{p}\right|^{2}
$$

Then, the unitary matrix $\mathbf{V}$ corresponding to minimum $J$ over fixed $h$ iterations is said to be an approximate joint diagonalizer [3]. Once $\hat{\mathbf{A}}$ is estimated, the sources $\hat{\mathbf{s}}$ can be estimated using the inverse of Eq. (1):

$$
\hat{\mathbf{s}}=\hat{\mathbf{A}}^{-1} \mathbf{x}
$$




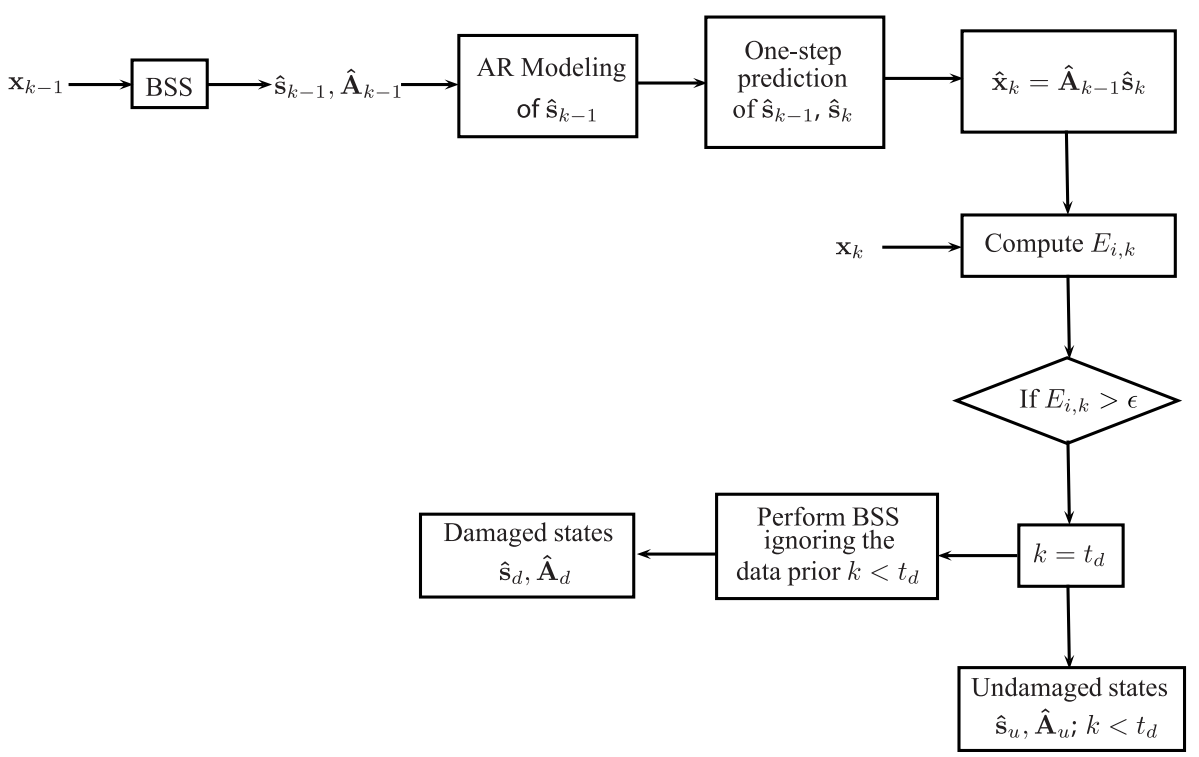

Fig. 4. Flowchart of the proposed method.

\subsection{Time-series modeling and one-step ahead prediction of sources}

Once the sources are extracted using the BSS method with the measurement up to $(k-1)^{\text {th }}$ time instant, the sources can be predicted for $k^{\text {th }}$ time instant using their time-series models. Since the sources are primarily monocomponent, they can be predicted using Eq. (6):

$$
\hat{\mathbf{s}}_{\mathbf{k}}=\phi_{1} \hat{\mathbf{s}}_{\mathbf{k}-1}+\phi_{\mathbf{2}} \hat{\mathbf{s}}_{\mathbf{k}-2}+\mathbf{a}_{\mathbf{t}}
$$

The AR parameters (i.e., $\phi(B))$ are estimated using sources up to $(k-1)^{\text {th }}$ time instant as described in Section 2. Once the sources are predicted, one-step ahead response can be estimated using:

$$
\hat{\mathbf{x}}_{\mathbf{k}}=\hat{\mathbf{A}}_{\mathbf{k}-\mathbf{1}} \hat{\mathbf{S}}_{\mathbf{k}}
$$

where $\hat{\mathbf{A}}_{\mathbf{k}-\mathbf{1}}$ is the mixing matrix based on the measurements up to $(k-1)^{\text {th }}$ time instant. With the occurrence of new measurement at $k^{\text {th }}$ time instant i.e, $\mathbf{x}_{\mathbf{k}}$, one can find the error between the predicted (i.e., $\hat{\mathbf{x}}_{\mathbf{k}}$ ) and true measurement $\left(\mathbf{x}_{\mathbf{k}}\right)$. Therefore, by utilizing a suitable error index, damage can be estimated using the true and predicted measurement. In this study, we define a variance-based error index as:

$$
E_{i, k}=\frac{R_{i}(0, k)-\hat{R}_{i}(0, k)}{R_{i}(0, k)}
$$

where, $R_{i}(0, k)=\sum_{n=0}^{k} \frac{x_{i}^{2}(n)}{k}$ and $\hat{R}_{i}(0, k)=\sum_{n=0}^{k} \frac{\hat{x}_{i}^{2}(n)}{k}$ are the variances of the true and the predicted measurement of $i^{\text {th }}$ floor location at $k^{\text {th }}$ time instant. When the error index $E_{i, k}$ attains a value more than a specified tolerance $\epsilon$, then it signifies that the modeling of $\hat{\mathbf{s}}$ based on the measurements up to $(k-1)^{\text {th }}$ time instant is inappropriate. This particular scenario happens when there are significant changes in the system and leads to a situation when the damage occurs. Under such situation, the measurements up to $(k-1)^{\text {th }}$ time instant represents the system response of undamaged state. On the other hand, the current measurements hereby represent the response of damaged state. Therefore the measurements up to $(k-1)^{\text {th }}$ time instant (i.e., damage instant $t_{d}$ ) is separated out and the BSS method is employed separately to undamaged and damaged data sets to estimate the modal parameters of undamaged $\left(\hat{s}_{u}, \hat{A}_{u}\right)$ and damaged system $\left(\hat{s}_{d}, \hat{A}_{d}\right)$ respectively. In this way, time series analysis-based error index is implemented in the framework of BSS to characterize the successive damage and undamaged states. The flowchart of the proposed method is shown in Fig. 4. 

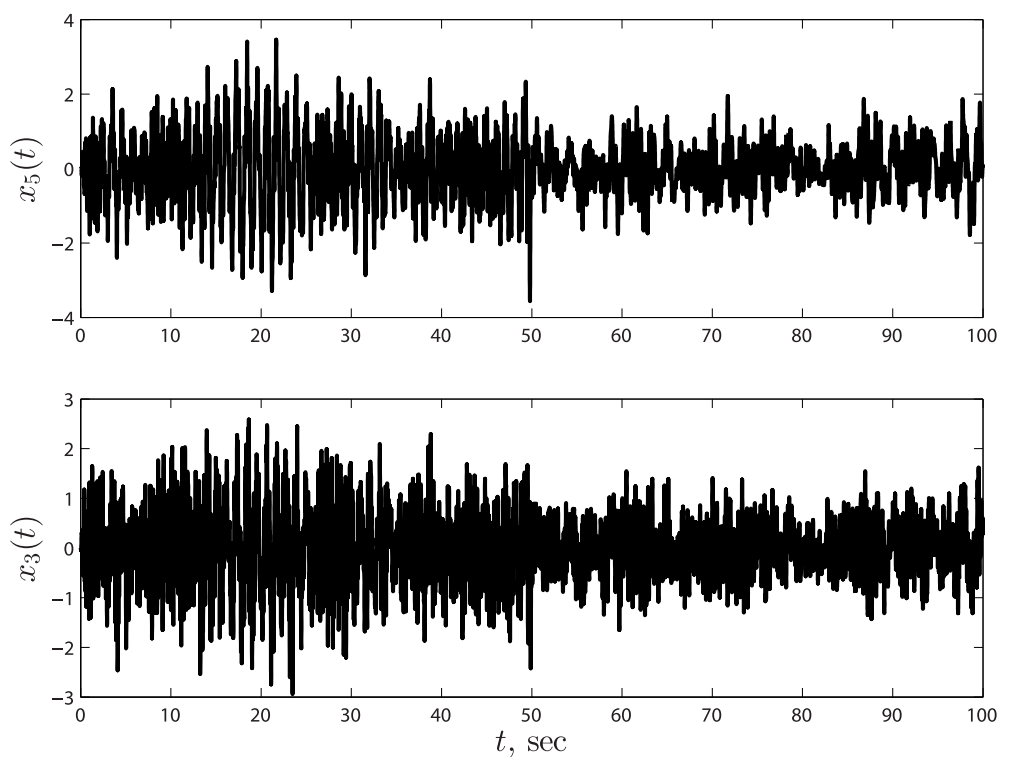

Fig. 5. Typical floor measurements showing the overall damage of the system at $t=50 \mathrm{sec}$.

\subsection{Identification of damage location}

Once we estimate the mode shapes of the damaged (i.e., $\hat{A}_{d}$ ) and undamaged system (i.e., $\hat{A}_{u}$ ), the damage location can be subsequently identified. It may be noted that the mode shape of the system under undamaged state can be utilized as a baseline data, and subsequently any difference in the mode shape ordinates under damaged state with respect to the baseline data (i.e., undamaged state) can be used to find the damage location. A new damage index is proposed herein to identify the differences in the mode shapes between the undamaged and damaged system as following:

$$
E_{i}^{\phi}=\frac{\sum_{j=1}^{n_{s}}\left\{\hat{A}_{d}^{i, j}-\hat{A}_{u}^{i, j}\right\}^{2}}{n_{s}}
$$

where $\hat{A}_{u}^{i, j}$ and $\hat{A}_{d}^{i, j}$ represent the $j^{\text {th }}$ mode shape ordinate at $i^{\text {th }}$ floor under undamaged and damaged states respectively and $E_{i}^{\phi}$ is the error index based on the mode shape ordinate (i.e., $\phi_{i}$ ) of the $i^{\text {th }}$ floor. The difference between the modal co-ordinates of the mode shapes under undamaged and damaged condition is used as performance index. Higher $E_{i}^{\phi}$ values amongst all floor levels indicate the damage location at any particular floor.

\section{Numerical simulation}

In order to illustrate an application of the proposed method, simulations are performed on a 5 -storey shear-beam structure model [29]. The state-space model for this system subjected to an external disturbance vector $\mathbf{w}$ is given by:

$$
\begin{aligned}
& \dot{\mathbf{x}}=\mathbf{A} \mathbf{x}+\mathbf{E} \mathbf{w} \\
& \mathbf{y}=\widetilde{\mathbf{C}} \mathbf{x}
\end{aligned}
$$

Here, the vector $\mathbf{x}$ is a vector of states, and the vector $\mathbf{y}$ represents the output vector, which is governed by $\widetilde{\mathbf{C}}$ matrix. The matrix $\mathbf{E}$ governs the location of the excitation on the structure. The system matrix $\mathbf{A}$ is constructed using $\mathbf{M}$, $\mathbf{C}$ and $\mathbf{K}$ matrices as follows.

$$
\mathbf{A}=\left[\begin{array}{cc}
\mathbf{0} & \mathbf{I} \\
-\mathbf{M}^{-1} \mathbf{K} & -\mathbf{M}^{-1} \mathbf{C}
\end{array}\right]
$$




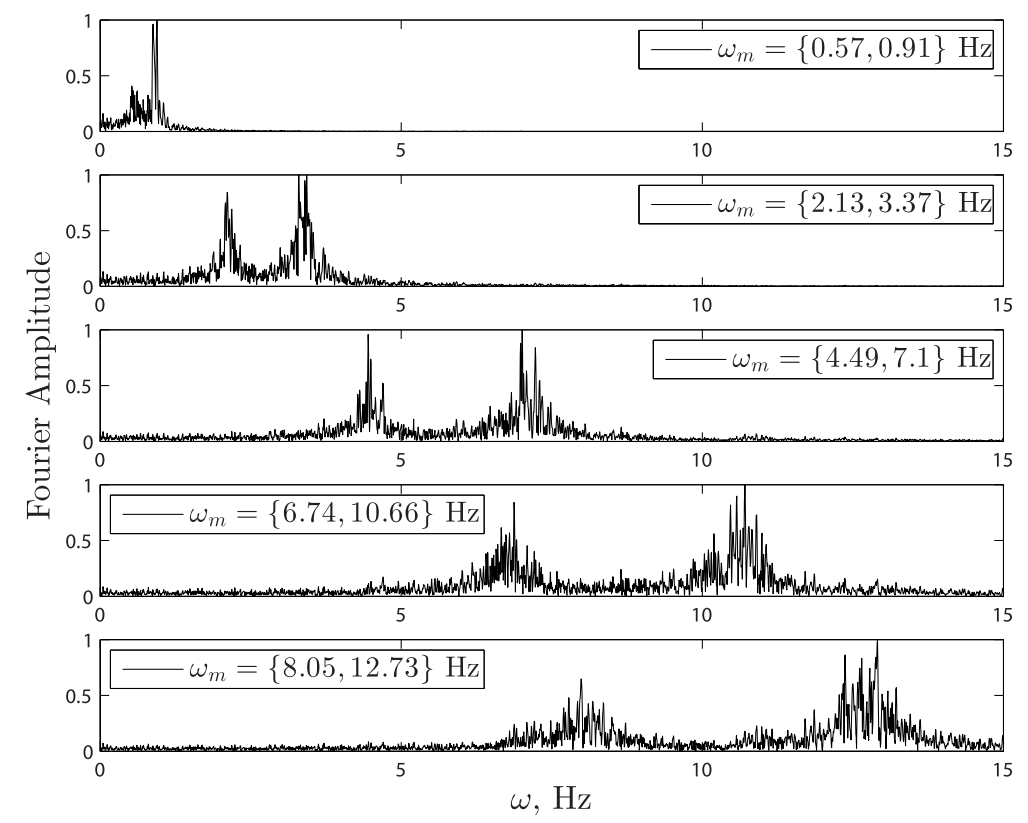

Fig. 6. Fourier spectra of the separated sources using SOBI method.

where $\mathbf{I}_{5 \times 5}$ and $\mathbf{0}_{5 \times 5}$ is an identity and zero matrix respectively. For the example building, the lumped weight of each floor is assumed to be $19.2 \mathrm{kN}$, and the damping is assumed to be $2 \%$ critical in all modes. The natural frequencies are $0.91,3.37,7.1,10.66$ and $12.73 \mathrm{~Hz}$. The mode shape matrix (normalized with respect to top floor) for the building is given by:

$$
\left[\begin{array}{rrrrr}
1.00 & 1.00 & 1.00 & 1.00 & 1.00 \\
0.82 & -0.087 & -1.29 & -2.52 & -3.39 \\
0.59 & -0.91 & -0.87 & 1.81 & 5.43 \\
0.34 & -1.02 & 1.23 & 0.94 & -5.84 \\
0.11 & -0.48 & 1.35 & -2.86 & 4.84
\end{array}\right]
$$

The correlation between the theoretical structural modes and the estimated modes is calculated using the modal assurance criterion (MAC) defined by [21]:

$$
M A C_{i}=\frac{\left(\psi_{i}^{T} \hat{\psi}_{i}\right)^{2}}{\left(\psi_{i}^{T} \psi_{i}\right)\left(\hat{\psi}_{i}^{T} \hat{\psi}_{i}\right)}
$$

where $\psi_{i}$ and $\hat{\psi}_{i}$ represent $i^{\text {th }}$ true and estimated mode shape vector respectively. MAC value of 1 indicates perfect correlation, and the values greater than 0.95 are usually considered good.

\subsection{Overall damage: Estimation of damage instant and modal parameters}

In order to simulate damage in the building, the overall stiffness is decreased by $60 \%$ at time $t=50$ seconds. The natural frequencies of the softened building are reduced to $0.57,2.13,4.49,6.74$ and $8.05 \mathrm{~Hz}$ respectively. Figure 5 shows $5^{\text {th }}$ and $3^{\text {rd }}$ floor responses of the damaged system, where there is a significant reduction in the floor response due to the softening of the system at $t_{d}=50$ seconds. The SOBI method [3] is employed using the floor measurements of 100 seconds including both the damaged and undamaged states. Equations (17) and (18) are used to estimate the mode shape and subsequently the sources are estimated using Eq. (19). Figure 6 shows the Fourier spectra of the separated sources. It is seen that there is presence of mode mixing $\left(f_{m}\right)$ in the frequency 


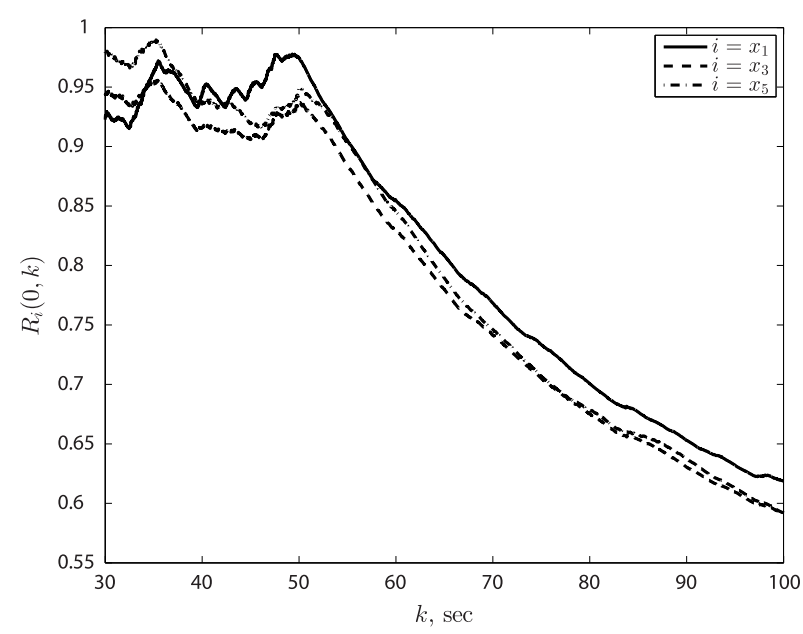

Fig. 7. Time-dependent variance of various floor measurements.

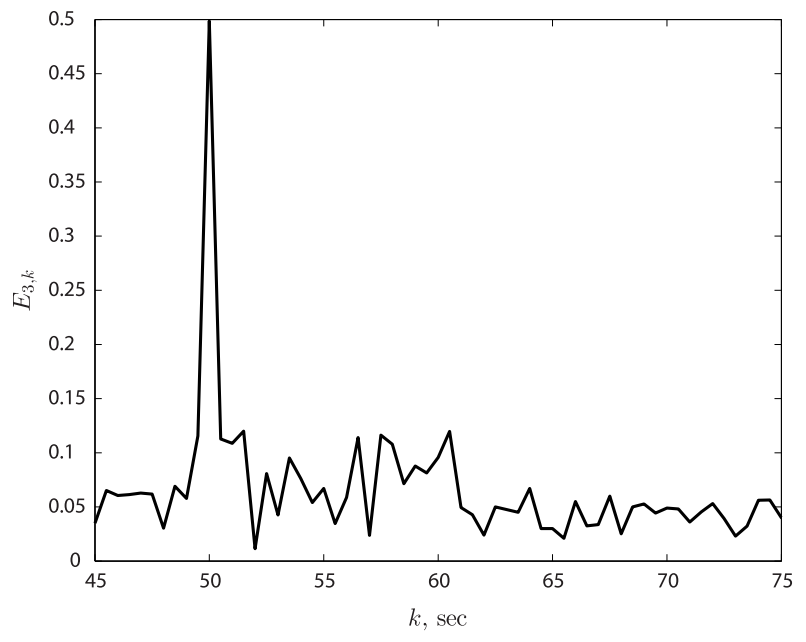

Fig. 8. Variation of $E_{3, k}$ in $x_{3}(t)$.

pairs of damaged and un-damaged building. For example, the first source contains the first natural frequency of the undamaged (i.e., $0.91 \mathrm{~Hz}$ ) and the damaged system (i.e., $0.57 \mathrm{~Hz}$ ) respectively. The estimation of mixing matrix becomes significantly erroneous due to presence of such mode mixing in the sources and yields the MAC numbers (see Eq. (27)) below 0.7. Therefore the standard SOBI method is unable to perform accurate source separation of the damaged and undamaged system in the current framework.

The proposed method is then employed in the floor vibration measurements. By looking at Fig. 5, it can be seen that the variance of the signal is almost constant before and after the damage. Figure 7 shows the time-dependent variances (i.e., $R_{i, k}$ ) of $1^{\text {st }}, 3^{\text {rd }}$ and $5^{\text {th }}$ floor respectively. There is a decaying nature in $R_{i, k}$ due to abrupt drop in the response envelope after the damage at $t_{d}=50$ seconds, which confirms the suitability of the variance based damage index as defined in Eq. (22).

Now, we use the proposed error index to identify the damage instant in adaptive fashion. Figure 8 shows the temporal variation of $E_{i, k}$ of the $3^{\text {rd }}$ floor measurement (i.e., $i=3$ ). It is seen that the error becomes extremely large at damage instant (i.e., $t_{d}$ ) due to inappropriate time series modeling of the predicted responses at time $t_{d}$ based on the un-damaged measurement as measured till time $t<t_{d}$. Such error index is utilized to ignore the measurements prior to time $t_{d}$ for the system identification of damaged state. When the measurements under undamaged state is separated out, the $E_{i, k}$ value is reduced. Immediately after the damage, initial fluctuation of $E_{i, k}$ is observed due to presence of fewer measurement data points of damaged data sets, however it gets stabilized with time when there is subsequent increase in the data length of newer measurements.

Figures 9 and 10 show the separated sources of the building for the un-damaged and damaged condition respectively. The advantage of the proposed method is that the sources in damaged and un-damaged states are obtained in an adaptive fashion for which the corresponding mixing matrices are estimated in an easier way. The mixing matrices of damaged and undamaged states are estimated using the sources of damaged and undamaged sources respectively. The MAC values are within 0.99 due to absence of the mode mixing in the respective sources. Therefore the presence of damage and its successive time instant is accurately identified, followed by the modal identification of the undamaged and damaged states.

So far the results are shown for an overall damage due to $60 \%$ decrease in the stiffness. In order to show the effect of damage in the performance of the proposed method, relatively broad range of stiffness reduction is considered which is characterized by a factor $\beta$. For example, $\beta=0.6$ means $60 \%$ reduction in overall stiffness. Table 2 summarizes the performance of the proposed method under different damage scenarios for a wide range of $\beta$. It is seen that the proposed method successfully detects the damage and identifies the modal parameters before and after the damage for $\beta=0.2-0.6$. However when $\beta=0.1$ (i.e., $10 \%$ reduction in overall stiffness), the natural frequencies of damaged and undamaged system are very close, and thus there is mode mixing in the resulting sources which yield poor MAC values. This is one of the limitations of the proposed method and is reserved for the future work. 

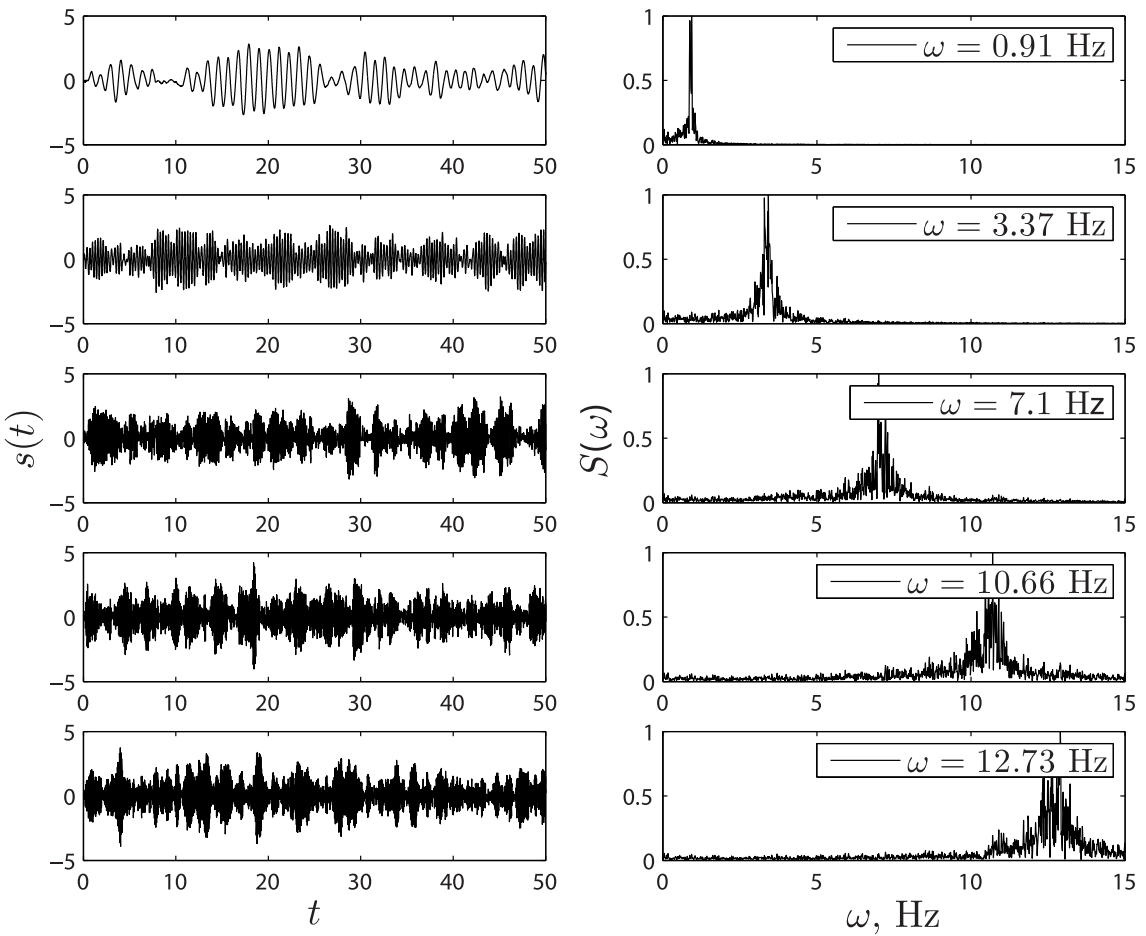

Fig. 9. Separated sources $\left(S_{u}\right)$ of undamaged system.
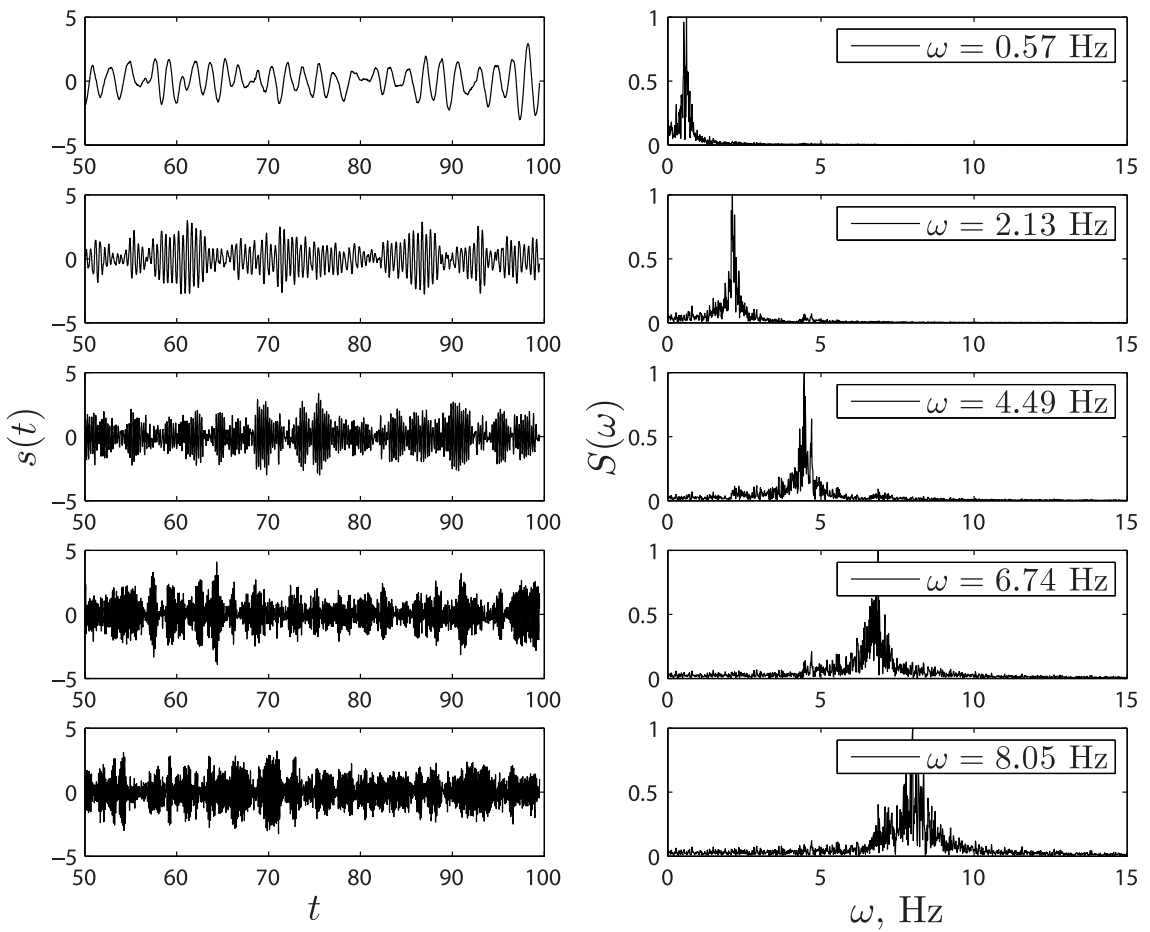

Fig. 10. Separated sources $\left(S_{d}\right)$ of damaged system. 
Table 2

Damage sensitivity of the proposed method

\begin{tabular}{lccccc}
\hline$\beta$ & 0.6 & 0.4 & 0.2 & 0.1 & 0.0 \\
\hline Damage detected & Yes & Yes & Yes & No & No \\
MAC (Before/After damage) & $1.0 / 1.0$ & $1.0 / 0.99$ & $1.0 / 0.99$ & $1.0 / 0.91$ & $1.0 / 0.99$ \\
\hline
\end{tabular}
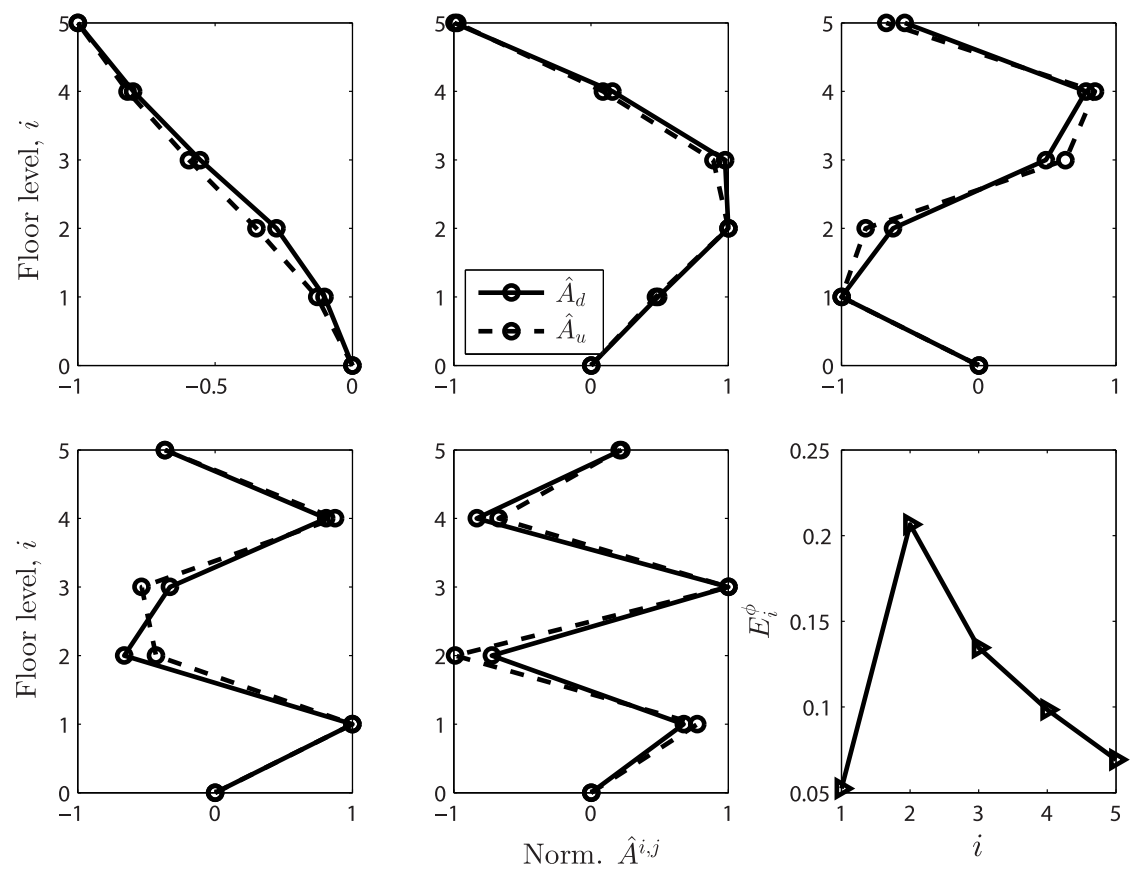

Fig. 11. Difference in mode shapes due to change in the inter-storey stiffness between $2^{\text {nd }}$ and $3^{\text {rd }}$ floor $(\alpha=25 \%)$.

\subsection{Elemental damage: Identification of damage location and its severity}

In this section, the proposed method is used to identify the location of damage within the building. For this purpose, the inter-storey stiffness between $2^{\text {nd }}$ and $3^{\text {rd }}$ floor is decreased by a factor $\alpha$. Figure 11 shows the estimated normalized mode-shapes under undamaged and damaged states using the proposed method and the plot of the error index (i.e., $E_{i}^{\phi}$ ) as defined in Eq. (23) with $\alpha$ of $25 \%$. It indicates that the highest error occurs in $2^{\text {nd }}$ floor followed by second highest error in $3^{\text {rd }}$ floor, which is evident from the fact that there is decrease in inter-story stiffness between $2^{\text {nd }}$ and $3^{\text {rd }}$ floor. Therefore the results clearly show that proposed error index using mode shapes can be used as an indicator of the damage locator. Figure 12 shows the variation in $E_{i}^{\phi}$ for various level of decreasing inter-storey stiffness between $2^{\text {nd }}$ and $3^{\text {rd }}$ floor. It can be seen that the $E_{i}^{\phi}$ increases significantly with the damage, especially around $2^{\text {nd }}$ and $3^{\text {rd }}$ floor.

\section{Full scale study}

In this section, the proposed identification method is applied to the ambient vibration data recorded during Parkfield earthquake at the UCLA Doris and Louis Factor building (UCLAFB) [20]. This building houses several centers for the health sciences and other biomedical facilities of UCLA. The structure is $74.5 \mathrm{~m}$ in height and consists of 15 floors plus a roof and a basement. The plan of this structure is rectangular with dimensions $30.5 \times 22.35 \mathrm{~m}$ [20].

This building is one of the most extensively instrumented buildings in North America, whose vibration data in real-time is made available for researchers world-wide through a remote data-base server. Designed and constructed in the late 1970s, this building consists of a special moment resisting steel frames (SMFs) supported by concrete 


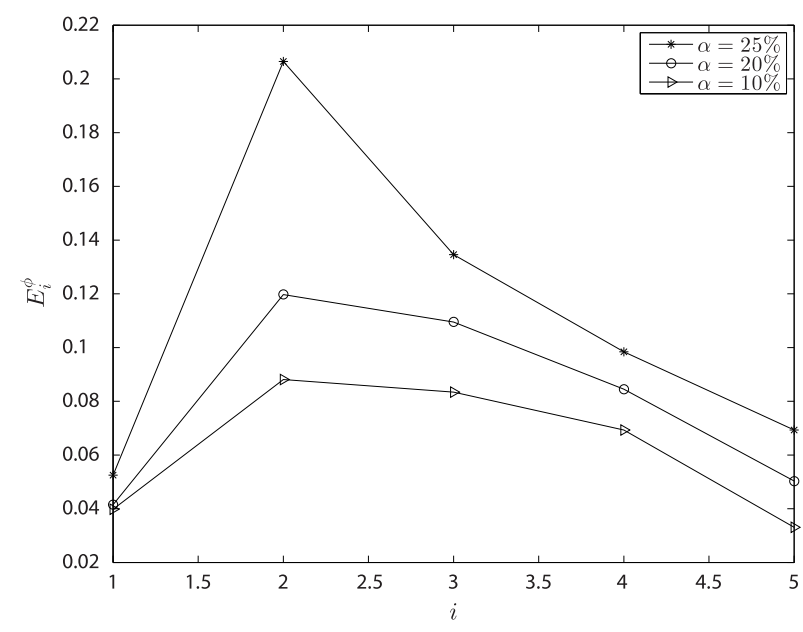

Fig. 12. Variation in RMS error in the mode shape coordinates at different floor levels.

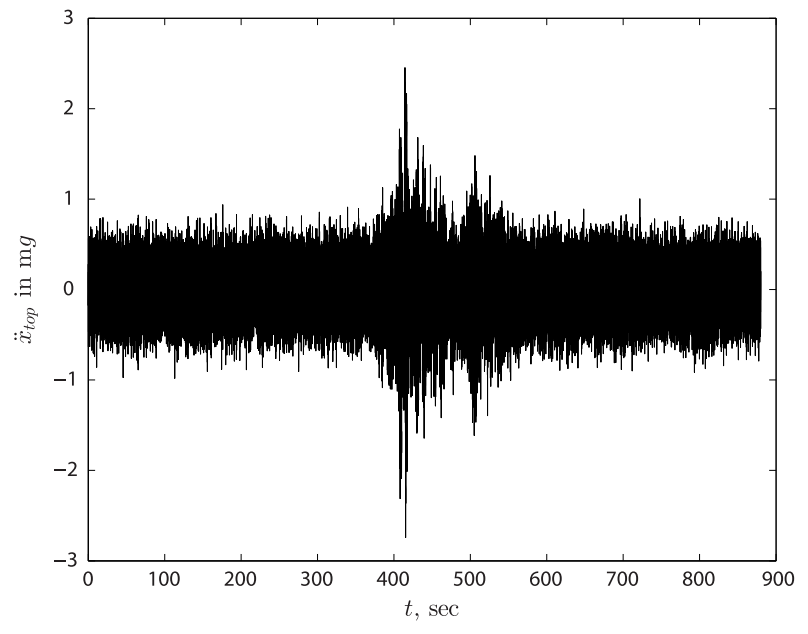

Fig. 13. Roof acceleration of UCLAFB in NS direction.

bell caissons and spread footings. Following the 1994 Northridge earthquake, the building was instrumented with an array of 72 Kinemetrics FB-11 uniaxial-accelerometers at the floor levels including the basement and the subbasement levels. Each level has two pairs of orthogonal sensors parallel to the NS and EW directions. The building's sensor network was upgraded in 2003 to a 24-bit network that continuously records data that includes numerous small earthquakes to date. Details of this building along with significant seismic events recorded by this network has been extensively documented in several published articles [10,20,23,29,31,33].

For the current identification study, the transformed floor accelerations (to the center of mass of the floors) recorded during Parkfield earthquake on September 28, 2004 at 10:15 AM PDT are used. Furthermore, consistent with the current set-up for the wired sensors at this building, each sensor is assumed to measure only one direction. Typical measured acceleration at roof is plotted in Fig. 13. Sudden jump in the roof acceleration at $t=400 \mathrm{sec}$ implies the occurrence of Parkfield earthquake. The earthquake with $M_{w}=6.0$ on the moment magnitude scale originated in Parkfield, CA, epi-centered 163 miles from the UCLAFB. The peak acceleration recorded at the roof of UCLAFB was $0.0025 \mathrm{~g}$.

The occurrence of Parkfield earthquake introduced damage in the building and softened the system. All the floor responses are used for identification using the proposed method. Typical plots of time-dependent variances of the floor responses at $1^{\text {st }}$ and $2^{\text {nd }}$ floor are shown in Fig. 14. The proposed method identifies the damage instant as $t_{d}=400 \mathrm{sec}$ which is detected using the proposed error index (see Eq. (22)) based on variance of the signal as shown in Fig. 14. Once the damage instant is identified, the first 400 seconds of ambient response data prior to the earthquake is then used for the identification of the undamaged state of the building and the data after $t_{d}=400$ sec is subsequently used for damage state estimation. The undamaged states and damaged states are identified using the measurements prior and after $t_{d}$ respectively. Tables 3 and 4 show the identification results, i.e., the modal frequency $(\omega)$ and damping $(\xi)$ of the undamaged and damaged system respectively for first 12 modes. The identification results using the proposed method are compared with the popular stochastic subspace identification (SSI) method [33], which is performed separately using the measurement of undamaged and damaged segments of the response. The estimated modal parameters compare significantly well with the SSI method. It may be noted that the use of computationally expensive stability diagrams prevents the use of SSI in adaptive algorithms. On the other hand, the proposed time-series based BSS method is free from user-interventions, and thereby extremely suitable for the real-time implementation. The results show that the proposed method leads to accurate estimation of damage instant and the successive identification of damaged and undamaged states. 
Table 3

Undamaged state: Identified $\omega$ and $\xi$ of the UCLAFB before the occurrence of Parkfield earthquake

\begin{tabular}{|c|c|c|c|c|c|}
\hline $\begin{array}{c}\text { Mode } \\
\text { No }\end{array}$ & $\begin{array}{l}\text { Shapes } \\
\text { Dir }\end{array}$ & $\begin{array}{c}\omega \\
\text { SSI }\end{array}$ & $\begin{array}{c}(\mathrm{Hz}) \\
\text { Proposed }\end{array}$ & $\begin{array}{c}\xi \\
\text { SSI }\end{array}$ & $\begin{array}{c}(\%) \\
\text { Proposed }\end{array}$ \\
\hline 1 & EW & 0.545 & 0.532 & 5.1 & 4.1 \\
\hline 2 & NS & 0.588 & 0.580 & 8.3 & 4.2 \\
\hline 3 & Torsion & 0.807 & 0.794 & 10.8 & 5.0 \\
\hline 4 & EW & 1.63 & 1.65 & 2.1 & 2.6 \\
\hline 5 & NS & 1.79 & 1.82 & 1.4 & 1.8 \\
\hline 6 & Torsion & 2.48 & 2.47 & 2.9 & 3.5 \\
\hline 7 & EW & 2.83 & 2.86 & 2.2 & 2.6 \\
\hline 8 & NS & 3.06 & 3.05 & 1.3 & 2.1 \\
\hline 9 & Torsion & 4.02 & 4.01 & 2.9 & 3.5 \\
\hline 10 & EW & - & 4.2 & - & 3.1 \\
\hline 11 & NS & - & 4.76 & - & 2.6 \\
\hline 12 & Torsion & - & 5.12 & - & 3.2 \\
\hline
\end{tabular}

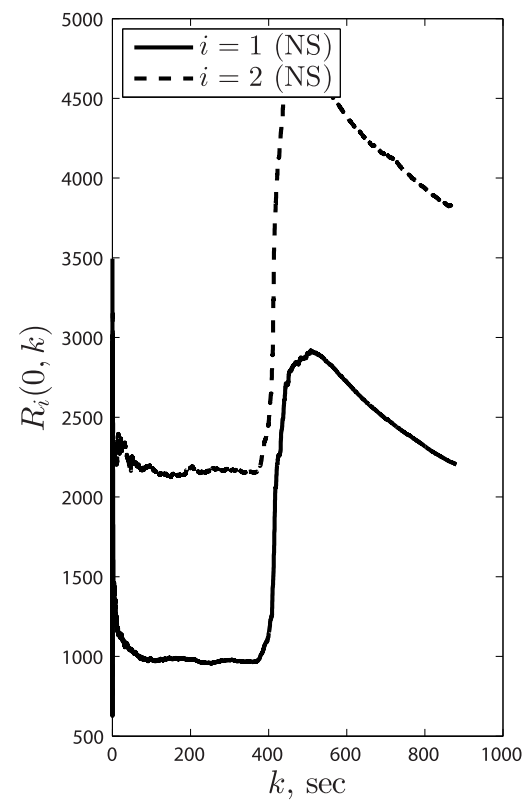

Table 4

Damaged state: Identified $\omega$ and $\xi$ of the UCLAFB after the occurrence of Parkfield earthquake

\begin{tabular}{|c|c|c|c|c|c|}
\hline \multirow{2}{*}{$\begin{array}{c}\text { Mode } \\
\text { no }\end{array}$} & \multirow{2}{*}{$\begin{array}{l}\text { Shapes } \\
\text { Dir }\end{array}$} & \multicolumn{3}{|c|}{$\omega(\mathrm{Hz})$} & \multirow{2}{*}{$\begin{array}{c}\xi(\%) \\
\text { Proposec }\end{array}$} \\
\hline & & SSI & Proposed & SSI & \\
\hline 1 & EW & 0.467 & 0.462 & 4.8 & 4.1 \\
\hline 2 & NS & 0.506 & 0.501 & 4.7 & 4.2 \\
\hline 3 & Torsion & 0.681 & 0.690 & 5.8 & 4.6 \\
\hline 4 & EW & 1.488 & 1.510 & 5.4 & 4.2 \\
\hline 5 & NS & 1.665 & 1.622 & 4.9 & 4.2 \\
\hline 6 & Torsion & 2.362 & 2.382 & 7.4 & 5.2 \\
\hline 7 & EW & 2.677 & 2.668 & 4.4 & 3.5 \\
\hline 8 & NS & 2.862 & 2.858 & 4.9 & 3.8 \\
\hline 9 & Torsion & 3.826 & 3.814 & 4.6 & 3.6 \\
\hline 10 & EW & - & 4.12 & - & 3.1 \\
\hline 11 & NS & - & 4.32 & - & 3.4 \\
\hline 12 & Torsion & - & 4.92 & - & 3.8 \\
\hline
\end{tabular}

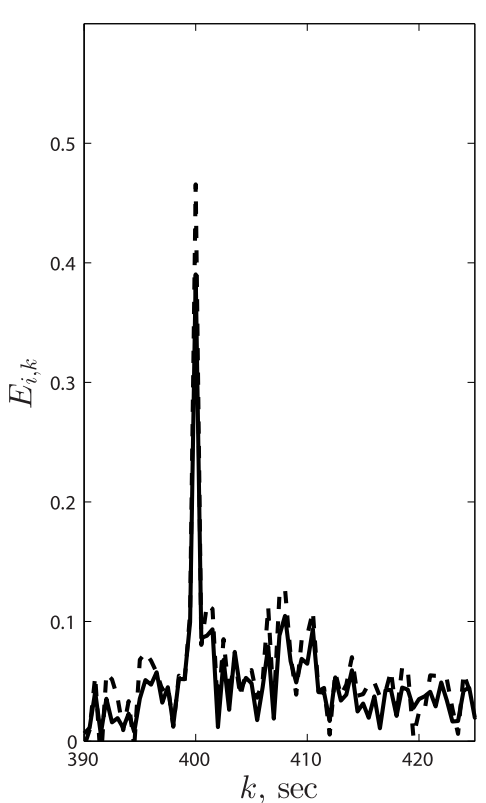

Fig. 14. Time-dependent variance and damage index of the typical floor response in NS direction.

\section{Conclusion}

In the present paper, blind source separation-based damage detection method in conjuction with time-series analysis is proposed for civil structures. Blind source separation is first utilized to estimate the modal responses using the vibration measurements. Time-series model is then employed for one-step ahead prediction of the past measurements. With the arrival of the newer measurements contaminated with the damaged state, the proposed error index is used to identify the damage instant. Once the damage instant is identified, the damage and undamaged modal parameters of the system are estimated in an adaptive fashion utilizing in the framework of blind source separation. Classical damage detection issues including identification of damage instant, location and severity of damage are addressed using the proposed method. The proposed method is verified via numerical simulations followed by the full scale study of UCLA factor building using ambient vibration data under Parkfield earthquake. 


\section{References}

[1] H. Akaike, A new look at the statistical model identification, IEEE Transactions on Automatic Control 19 (1974), $716-723$.

[2] J. Antoni, Blind separation of vibration components: Principles and demonstrations, Mechanical Systems and Signal Processing 19 (2005), 1166-1180.

[3] A. Belouchrani, K. Abed-Meraim, J. Cardoso and E. Moulines, A blind source separation technique using second-order statistics, IEEE Transactions on Signal Processing, IEEE 45(2) (1997), 434-444.

[4] J.B. Bodeux and J.C. Golinval, Application of armav models to the identification and damage detection of mechanical and civil engineering structures, Smart Materials and Structures 10 (2001), 479-489.

[5] G.E.I. Box, G.M. Jenkins and G.C. Reinsel, Time Series Analysis: Forecasting and Control, John Wiely and Sons, Hoboken, New Jersey, 2008.

[6] E.P. Carden and P. Fanning, Vibration based condition monitoring: A review, Structural Health Monitoring 3(4) (2004), 355-377.

[7] A. Cheung, C. Cabrera, P. Sarabandi and A.W.H. Kiremidjian, The application of statistical pattern recognition methods for damage detection to field data, Smart Materials and Structures, 2008.

[8] A. Cichocki and S. Amari, Adaptive Blind Signal and Image Processing, John Wiley and Sons, Ltd., Wiley (West Sussex), UK, 2003.

[9] S. Doebling, C. Farrar, M. Prime and D. Shevitz, Damage identification and health monitoring of structural and mechanical systems from changes in their vibration characteristics: A literature review, Technical Report LA-13070-MS, Los Alamos National Laboratory, Los Alamos, NM, 1996.

[10] B. Hazra and S. Narasimhan, Wavelet-based blind identification of the UCLA Factor building using ambient and earthquake responses, Smart Materials and Structures 19(2) (2010), 025005.

[11] B. Hazra, A.J. Roffel, S. Narasimhan and M.D. Pandey, Modified cross-correlation method for the blind identification of structures, Journal of Engineering Mechanics, ASCE 136(7) (2010), 889-897.

[12] B. Hazra, A. Sadhu, A.J. Roffel and S. Narasimhan, Hybrid time-frequency blind source separation towards ambient system identification of structures, Computer Aided Civil and Infrastructure Engineering 27(5) (2012), 314-332.

[13] B. Hazra, A. Sadhu, R. Lourenco and S. Narasimhan, Retuning tuned mass dampers using ambient vibration response, Smart Materials and Structures, IOP Publishing 19(11) (2010), 115002.

[14] B. Hazra, A. Sadhu, A.J. Roffel, P.E. Paquet and S. Narasimhan, Underdetermined blind identification of structure by using the modified cross-correlation method, Journal of Engineering Mechanics, ASCE 138(4) 2012.

[15] K.W. Hipel and A.I. Mcleod, Time Series Modelling of Water Resources and Environmental Systems, Elsevier, Amsterdam, Netherlands, 1994.

[16] Z. Hou, M. Noori and R.S. Amand, Wavelet-based approach for structural damage detection, J Engrg Mech ASCE 126(7) (2000).

[17] A.K.J. Hyvarinen and E. Oja, Independent Component Analysis, John Wiley, New York, 2001.

[18] J. JianPing and M. Guang, A novel method for multi-fault diagnosis of rotor system, Mechanism and Machine Theory 44 (2009), $697-709$.

[19] G. Kerschen, F. Poncelet and J. Golinval, Physical interpretation of independent component analysis in structural dynamics, Mechanical Systems and Signal Processing 21 (2007), 1561-1575.

[20] M.D. Kohler, M.P. Davis and E. Safak, Earthquake and ambient vibration monitoring of the steel-frame UCLA Factor building, Earthquake Spectra, Earthquake Engineering Research Institute 21(3) (2005), 1-22.

[21] N.M.M. Maia and J.M.M. Silva, Theoretical and Experimental Modal Analysis, Research Studies Press, UK, 1997.

[22] K.K. Nair, A.S. Kiremidjian and K.H. Law, Time series-based damage detection and localization algorithm with application to the asce benchmark structure, Journal of Sound and Vibration 291 (2006), 349-368.

[23] R.D. Nayeri, S.F. Masri, R. Ghanem and R.L. Nigbor, A novel approach for the structural identification and monitoring of a full-scale 17-story building based on ambient vibration measurements, Smart Materials and Structures 17(2) (2008), 1-19.

[24] J.S. Owen, B.J. Eccles, B.S. Choo and M.A. Woodings, The application of autoregressive time series modelling for the timefrequency analysis of civil engineering structures, Engineering Structures 23 (2001), 521-536.

[25] S.M. Pandit and S.M. Wu, Time Series and System Analysis with Applications, John Wiley and Sons, New York, 1983.

[26] R. Rodriguez, J.A. Escobar and R. Gomez, Damage detection in instrumented structures without baseline modal parameters, Engineering Structures 32 (2010), 1715-1722.

[27] C. Rutten, V.H. Nguyen and J.C. Golinval, Comparison of output-only methods for condition monitoring of industrials systems, 9th International Conference on Damage Assessment of Structures: Journal of Physics 305 (2011). doi:10.1088/1742-6596/305/1/012101.

[28] A. Rytter, Vibration based inspection of civil engineering structures, Ph.D. Dissertation, Department of Building Technology and Structural Engineering, Aalborg University, Denmark, 1993.

[29] A. Sadhu, B. Hazra, S. Narasimhan and M.D. Pandey, Decentralized modal identification using sparse blind source separation, Smart Materials and Structures, IOP Publishing 20(12) (2011), 125009.

[30] A. Sadhu and S. Narasimhan, Blind source separation of convolutive mixtures towards modal identification, Conference Proceedings of the Society for Experimental Mechanics Series (26) (2012), 209-220. DOI: 10.1007-978-1-4614-2413-0-21.

[31] A. Sadhu, B. Hazra and S. Narasimhan, Blind identification of earthquake-excited structures, Smart Materials and Structures, IOP Publishing 21(4) (2012), 045019.

[32] A. Sadhu, B. Hu and S. Narasimhan, Blind source separation towards decentralized modal identification using compressive sampling, Proceedings of the $11^{\text {th }}$ International Conference on Information Science, Signal Processing and their Applications by IEEE: Special Sessions, Montreal, Canada (2012) 1184-1189. DOI: 978-1-4673-0382-8-12.

[33] D. Skolnik, Y. Lei, E. Yu and J. Wallace, Identification, model updating, and response prediction of an instruemented 15-story steel-frame building, Earthquake Spectra, Earthquake Engineering Research Institute 22(3) (2006), 781-802.

[34] W.J. Staszewski and A.N. Robertson, Time-frequency and time-scale analyses for structural heal monitoring, Philosophical Transction of The Royal Society 365 (2007), 449-477. 
[35] A.M. Yan, G. Kerschen, P.D. Boe and J.C. Golinval, Structural damage diagnosis under varying environmental conditions-part i: A linear analysis, Mechanical Systems and Signal Processing 19 (2005), 847-864.

[36] Y.J. Yan, L. Cheng, Z.Y. Wu and L.H. Yam, Development in vibration-based structural damage detection technique, Mechanical Systems and Signal Processing 19 (2007), 2198-2211.

[37] J.N. Yang, Y. Lei, S. Lin and N. Huang, Hilbert-huang based approach for structural damage detection, Journal of Engineering Mechanics, ASCE 130(1) (2004), 85-95.

[38] R. Yao and S.N. Pakzad, Autoregressive statistical pattern recognition algorithms for damage detection in civil structures, Mechanical Systems and Signal Processing 31 (2012), 355-368.

[39] C. Zang, M.I. Friswell and M. Imregun, Structural damage detection using independent component analysis, Structural Health Monitoring 2 (2004), 69-83. 

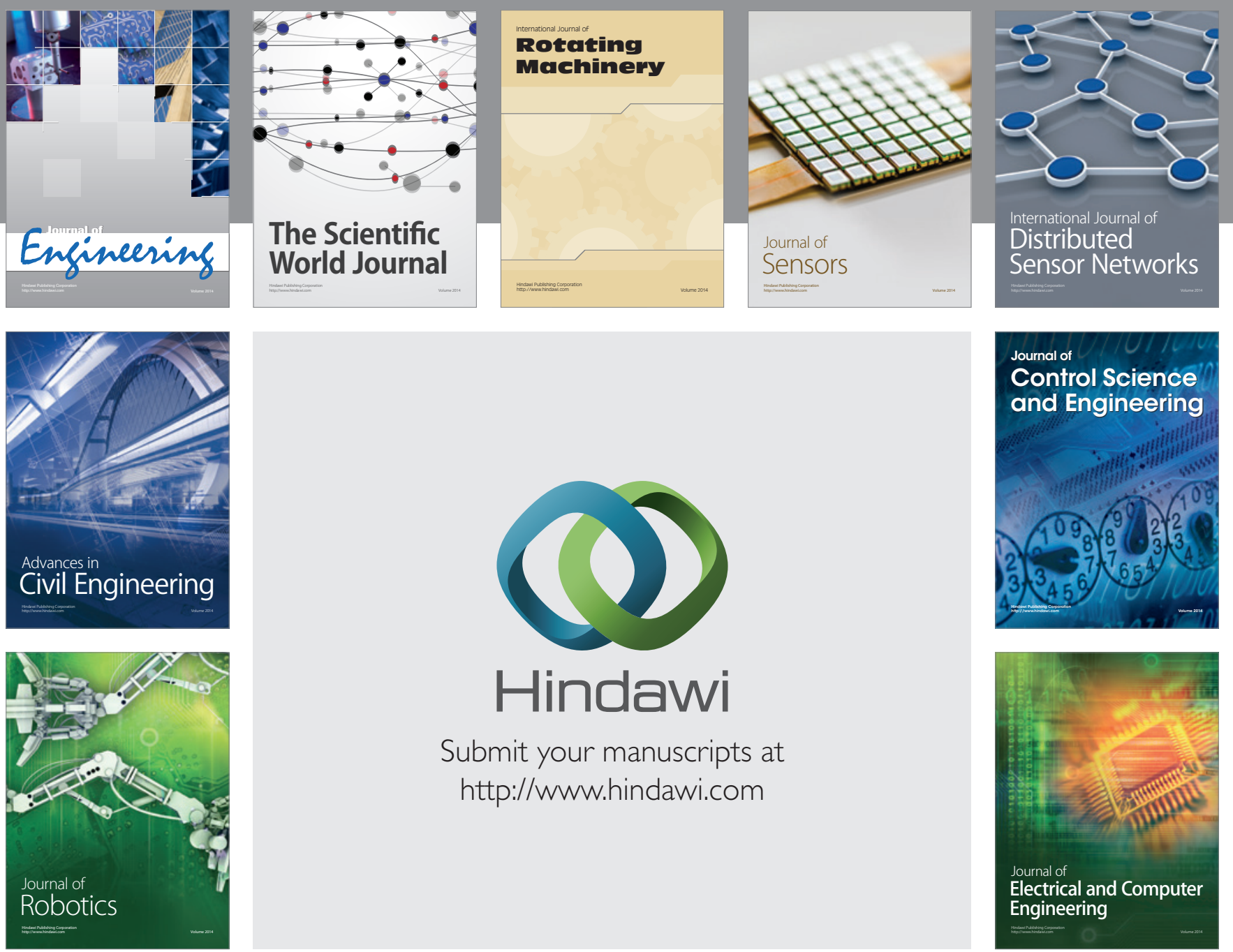

Submit your manuscripts at

http://www.hindawi.com
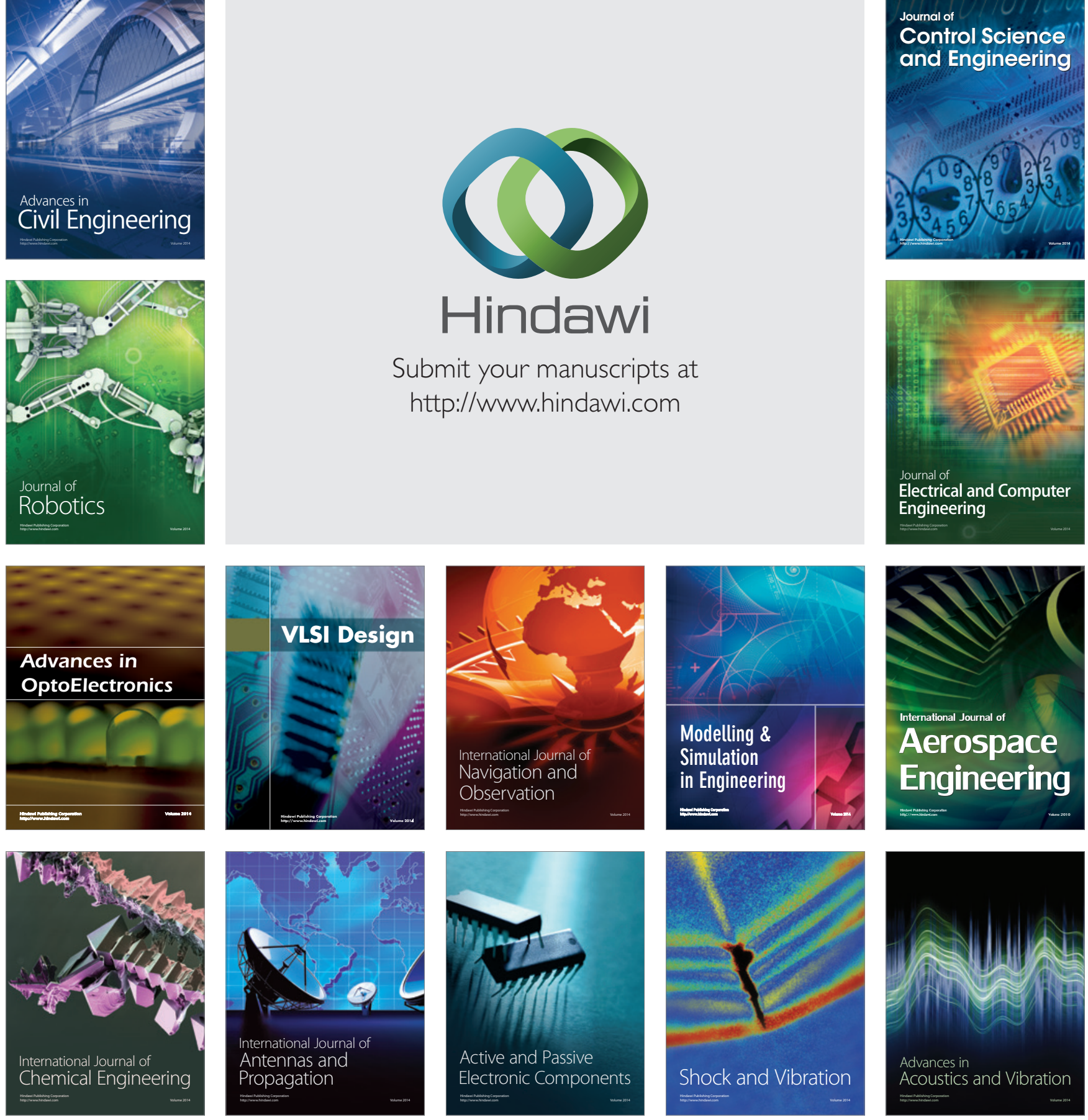